\title{
La adopción de la tecnología cloud computing (SaaS): efectos de la complejidad tecnológica vs formación y soporte
}

\author{
Pedro Ramiro Palos-Sánchez ${ }^{1}$, Francisco J. Arenas-Márquez ${ }^{2}$, Mariano Aguayo- \\ Camacho $^{2}$
}

ppalos@unex.es, fjarenas@us.es, maguayo@us.es

${ }^{1}$ Departamento de Dirección de Empresas y Sociología, Universidad de Extremadura - España

${ }^{2}$ Departamento de Economía Financiera y Dirección de Operaciones, Universidad de Sevilla - España

DOI: DOI: 10.17013/risti.22.89-105

Resumen: El propósito de este trabajo es analizar empíricamente, la influencia de las variables Complejidad Tecnológica, Formación y Soporte y Apoyo Técnico en el seno de una organización y como pueden afectar a la intención de uso final de la tecnología cloud. Se evaluaron, a través de una encuesta, 150 empresas de Andalucía. Los aspectos claves que se han identificado con dicha intención han sido la disponibilidad en el soporte técnico, dudas o problemas de uso relacionados con la complejidad y la adecuación de la formación recibida por parte de la organización. Los resultados indican que la complejidad ejerce un efecto negativo, mientras que la formación y el soporte son claves, con un peso muy similar, en la intención de uso, descubriéndose que el conocimiento compartido o cooperativo es clave en la adopción de la tecnología cloud computing.

Palabras-clave: Cloud Computing; SaaS; Complejidad Tecnológica; Formación Profesional; Soporte y Apoyo Técnico.

The adoption of cloud computing technology (SaaS): effects of technological complexity $v$ s training and support

\begin{abstract}
The purpose of this paper is to analyze empirically the influence of the variables Technological Complexity, Training and Support and Technical Support within an organization and how they can affect the end use intention of cloud technology. 150 companies from Andalusia were evaluated through a survey. The key aspects that have been identified with this intention have been the availability in the technical support, doubts or problems of use related to the complexity and the adequacy of the training received by the organization. The results indicate that complexity has a negative effect, while training and support are key, with a very similar weight, in the intention to use, finding that shared or cooperative knowledge is key in the adoption of cloud computing technology.
\end{abstract}

Keywords: Cloud Computing; SaaS; Technological Complexity; Training; Cooperation and Technical Support. 


\section{Introducción}

El cloud computing o computación en la nube, nace de los términos: cloud y computing (Torres, 2011). Cloud, o nube, es el símbolo que se usa generalmente para representar Internet. Se establece un concepto de abstracción (sistemas físicos que no se especifican, almacenamiento de datos en ubicaciones desconocidas, acceso ubicuo de los usuarios y administraciones subcontratadas). Y Computing o computación, reúne los conceptos de informática, lógica de coordinación y almacenamiento.

El uso del término "nube" es metafórico y por lo general apunta a un gran conjunto de recursos disponibles, hardware y software, que son de fácil acceso a través de Internet (Sá \& Rocha, 2012; Vaquero et al., 2009; Vouk, 2008). Para el Instituto Nacional de Estándares y Tecnología de los Estados Unidos de América (Mell \& Grance, 2011) el cloud computing es un modelo para habilitar el acceso a un conjunto de servicios computacionales (redes, servidores, almacenamiento, aplicaciones y servicios) de manera conveniente y por demanda, que pueden ser rápidamente aprovisionados y liberados con un esfuerzo administrativo y una interacción con el proveedor de servicio mínima (Forrester Research, 2009; Gartner, 2008; Buyya et al., 2009).

La tecnología cloud supone un avance, pero su adopción debe hacerse bajo un esquema modular y que ofrezca grandes facilidades de reconfiguración, en el que se puede flexiblemente acumular recursos o servicios que satisfagan las cambiantes exigencias del mercado. No obstante, el concepto aún se encuentra en España en particular y globalmente en general en su etapa más incipiente de adopción afectando de forma dispar a los dos sectores clave del tejido empresarial: la gran empresa y la pequeña y mediana empresa (Pyme). Para que la computación en la nube pueda crecer, es importante comprender los factores que pueden influir en su tasa de adopción por parte de las organizaciones, especialmente empresas. Conocer la influencia que la complejidad, la formación en sistemas cloud al personal de la organización y recibir soporte y apoyo de la organización en el proceso de adopción, es el objetivo de este trabajo, el cual está estructurado en un primer apartado llamado marco teórico, que justifica la elección de estas tres variables. Le sigue un apartado de análisis, a través de ecuaciones estructurales, del modelo estructural. Este artículo finaliza contrastando las hipótesis y presentando las conclusiones alcanzadas.

\section{Marco teórico}

Se identificaron las investigaciones más relevantes sobre los sistemas cloud y sus modelos de adopción en función de los objetivos de este artículo. Así, Burda \& Teuteberg et al. (2014) afirman que, con frecuencia, la literatura tecnológica se centra en abordar cuestiones como la seguridad de la infraestructura cloud mediante la propuesta de nuevas arquitecturas y métodos (véase, por ejemplo, Spillner et al., 2011; Brandt et al., 2012; Wang et al., 2013). En otros casos, el eje principal de investigaciones son cuestiones relacionadas con oportunidades, costes y riesgos asociados al cloud computing (Ackermann et al, 2013; Benlian \& Hess, 2011; Martens \& Teuteberg, 2012; Palos-Sanchez et al., 2017a; Palos \& Correia, 2016), criterios de medición de la calidad del servicio (Benlian et al., 2011) o factores relacionados con la adopción SaaS (PalosSanchez et al., 2017b), como la disponibilidad del servicio, la accesibilidad (Palos \& 
Correia, 2017), su rendimiento, la falta de estándares de interoperabilidad y su dificultad de integración y personalización (Feuerlicht \& Govardhan, 2010; Feuerlicht et al, 2011; Géczy et al., 2012) o la influencia de los esfuerzos de marketing (Lin et al., 2010;Fortes et al., 2016). También es posible encontrar varios trabajos que inciden en la importancia de la confianza, tanto en la adopción de la tecnología cloud (Pearson, 2011; Walterbusch et al., 2013), como en las condiciones de privacidad en el almacenamiento de datos (Ion et al., 2011).

Otras investigaciones concluyen con la relevancia de aspectos tales como la incertidumbre, la compatibilidad, el apoyo de la alta dirección, la utilidad percibida, la facilidad de uso de la tecnología, la experiencia previa, las restricciones geográficas, el tamaño de la empresa, el mercado, los esfuerzos de los proveedores, la seguridad, la confianza, la influencia social y la presión de socios comerciales en la adopción de cloud computing (Alshamaila et al, 2013; Gangwar et al., 2015; Lin \& Chen, 2012; Low et al, 2011; Wu, 2011).

La introducción de intención de uso (IU) como variable final es frecuente, ya que anteriores estudios piensan que una intención siempre precede a un comportamiento y se aumenta así el valor predictivo del modelo (Fishbein \& Ajzen, 1975). Así, IU es el grado de comportamiento previo que se tiene a la hora de usar dicha tecnología (Taylor \& Todd, 1995).

Nuestro trabajo se basa también en investigaciones anteriores sobre sistemas de información ERP que destacan la importancia de la complejidad tecnológica o la formación (Bueno y Salmerón, 2008) y en estudios recientes sobre cloud de especial interés para la presente investigación (Gangwar et al., 2015), que entre otras variables destaca la formación, la complejidad y el apoyo externo como claves en la adopción y uso de la tecnología.

Por complejidad tecnológica (CT) entenderemos el grado a través del cual una tecnología es percibida como relativamente difícil para comprender y usar (Rogers \& Shoemaker, 1971). Algunos trabajos de investigación localizados en la literatura y relacionados con la complejidad, son el grado de dificultad de uso de las habilidades necesarias para utilizar las tecnologías entre los empleados o sobre la dificultad de integración de estas tecnologías en el trabajo (Premkumar \& Roberts, 1999).

Tomando como base dichos conceptos, podemos afirmar que existe una relación negativa entre la complejidad percibida y la utilización del cloud, para la cual intentaremos hallar evidencias que ratifiquen esta relación. Se asocia negativamente con la intención de uso (Cooper \& Zmud, 1990; Grover, 1993), ya que se percibe complejidad tecnológica en la proliferación de normas y protocolos para la correcta implementación de hardware y software en Sistemas de Información. Por lo tanto, la complejidad tecnológica puede actuar como una barrera para la intención de uso del cloud computing, confirmando la significación de dicha relación (Oliveira \& Martins, 2010; Wang et al., 2013). Así cuanto menor sea la influencia de la complejidad más probable será la intención de uso (Premkumar \& Roberts, 1999). En base a todo esto podemos proponer que:

H1: La complejidad tecnológica en sistemas cloud ejerce un efecto negativo sobre la intención de uso. 
La formación profesional (FP) se describe como el grado en el que una empresa instruye a sus empleados en el uso de una herramienta en términos de calidad y cantidad (Schillewaert et al., 2005). Dado que el cloud computing es un sistema de información complejo, una organización necesita entrenar y enseñar la aplicación de gestión cloud a sus empleados antes de su uso. Esto reduce la ansiedad y el estrés de los empleados en el uso del cloud, y proporciona la motivación y un mejor entendimiento acerca de sus beneficios para sus tareas. Además reduce la ambigüedad y ayuda a los empleados en el desarrollo de conocimientos para su uso eficaz en el futuro (Gangwar el al., 2015).

La formación en sistemas de información ayuda a los usuarios a familiarizarse con la herramienta, reduciendo los posibles obstáculos que se pudiesen derivar de la complejidad tecnológica (Amoako-Gyampah \& Salam, 2004; Cheung \& Vogel, 2013; Lee \& Lehto, 2013). Igualmente, la formación permite compartir problemas comunes entre los usuarios, circunstancia que pudiera tener el efecto de incrementar la comunicación vinculada al cloud. Este hecho se ha observado en otras investigaciones con sistemas de información, como en el caso de software ERP (Bueno y Salmerón, 2008). Por tanto se propone que:

H2: La formación ejerce un efecto positivo sobre la intención de uso de los sistemas cloud.

El soporte y apoyo en la organización (SA) se identifica un factor relevante en la adopción de sistemas de información. El Soporte y Apoyo es crucial, ya sea en su vertiente interna o externa, proporciona sinergia, da seguridad a los usuarios y ayuda al alcance de las expectativas (Joo \& Sang, 2013; Lee \& Lehto, 2013; Somers, 2003). El soporte y ayuda interna hace referencia a aquella que es ejercida entre las distintas áreas funcionales

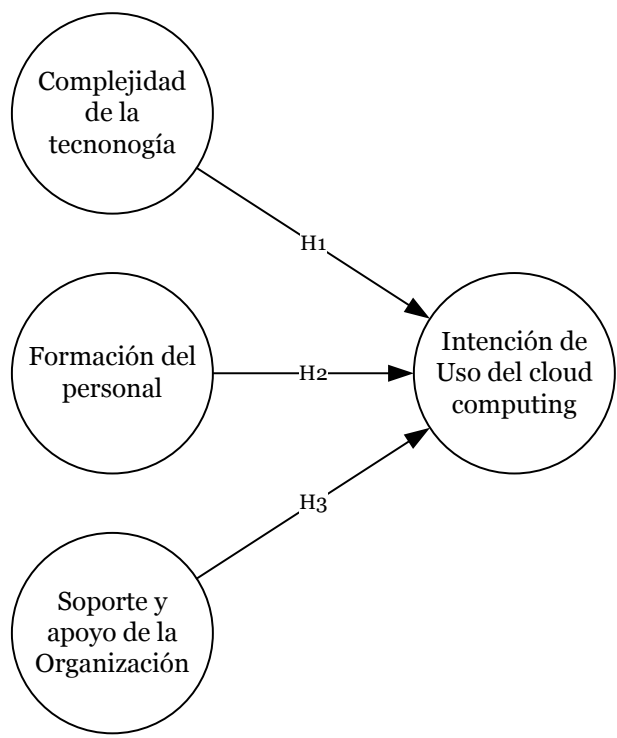

Figura 1 - Modelo teórico propuesto 
de una empresa. Por otra parte, el soporte y ayuda externa alude a los vínculos que mantiene la organización con el proveedor del cloud. En base a los razonamientos expuestos, queremos hallar evidencias para poner de manifiesto que la cooperación se encuentra relacionada positivamente con la percepción de utilidad del cloud (Cheung \& Vogel, 2013; Son et al., 2012).

En este sentido, suponemos que el soporte y apoyo y el tratamiento conjunto de los problemas derivados del cloud faciliten la intención de uso por parte de los usuarios. Esta proposición nos permite formular la siguiente hipótesis:

H3: El soporte y apoyo ejerce un efecto positivo sobre la intención de uso de los sistemas cloud.

La siguiente figura 1, representa el modelo planteado con las diferentes hipótesis:

\section{Metodología}

La población objeto de estudio en esta investigación, son todas aquellas empresas e instituciones que en Andalucía usan o se ven beneficiadas de la tecnología cloud computing en sus diferentes vertientes de forma regular, constituyendo ésta una tecnología estratégica en sus servicios. Por tanto, para poder ser parte de la población objeto de estudio se deben cumplir estas condiciones. El tamaño del universo poblacional de esta investigación es conocido, ya que hemos elaborado, en colaboración con diferentes organizaciones del sector, un censo de las empresas y organizaciones que ya trabajan con tecnologías cloud.

La técnica cuantitativa de investigación que se ha llevado a cabo ha sido la encuesta, materializada en un cuestionario online basado en preguntas de adopción aplicadas a otros sistemas de información y en particular a la tecnología cloud. Las preguntas se presentaron en un sitio web cuyo enlace fue enviado a la población objeto de estudio, donde respondió el representante legal o en quién éste delegara, con independencia del cargo desempeñado. La supervisión telefónica permitió que el cuestionario fueran respondido por la persona con mayor conocimiento del proceso de adopción del cloud dentro de la organización o directamente por la dirección, bajo la tipología de muestreo no probabilístico por conveniencia. Se obtuvieron un total de 161 cuestionarios, de los que fueron finalmente válidos para esta investigación 150. Participaron 13 sectores profesionales distintos dentro de los cuales hay que distinguir 2 que no son empresariales. Estos son Administración Pública y Organizaciones sin ánimo de lucro, como Organizaciones Empresariales o Colegios Profesionales. En este apartado hay que decir que destaca con fuerza el sector servicios con un 48,7\%, , seguido del sector TIC con un $22 \%$ y el resto está muy atomizado, destacando Educación con un 7,3\% o Construcción con un $4 \%$ al igual que Consultoría.

\section{Análisis de los resultados}

Para llevar a cabo este apartado de la tarea de investigación se ha seguido la metodología recogida en diferentes trabajos de Cepeda y Roldán (2004). Se ha elegido el método de análisis PLS (Partial Least Squares), ya que está especialmente recomendado para la 
investigación exploratoria y permite modelar constructos latentes tanto con indicadores formativos como reflectivos (Son y Benbasat, 2007). También, se ha elegido porque se han encontrado un significativo número de trabajos relacionados con la adopción del cloud computing, utilizando PLS e indicadores reflectivos en su mayoría, dentro de la escasez de trabajos existente hoy sobre cloud (Messerschmidt, 2013; Gupta et al, 2013; Senk, 2013).

La evaluación del modelo implica, analizar la fiabilidad y validez de las medidas utilizadas para representar cada constructo (Chin W., 2010). El objetivo de la fiabilidad individual es medir las cargas $(\lambda)$ del indicador, situando el nivel mínimo para aceptar el indicador como parte del constructo en $\lambda>=0,707$ (Carmines \& Zeller, 1979). Como

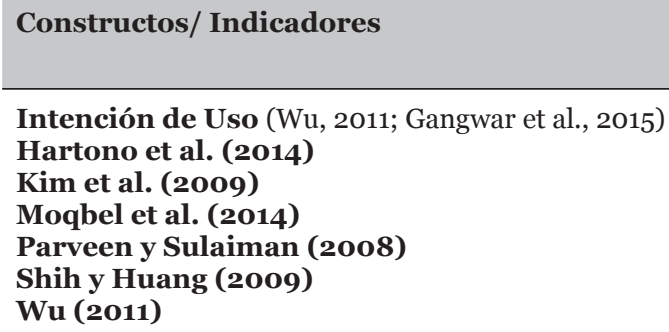

(IU1) Espero usar el sistema cloud computing. $\quad 0,893$

(IU2) Espero que la información del nuevo sistema de cloud computing sea útil. $\quad$ 0,93

Complejidad tecnológica (Bueno \& Salmerón, 2008; Gangwar et al., 2015)

(CT1) Es difícil comprender lo que hace el sistema de cloud computing.

(CT2) Usar el sistema de cloud computing me ocupa demasiado tiempo.

(CT3) Necesito mucho esfuerzo para aprender a usar el sistema cloud computing.

0,922

(CT4) En general, el sistema de cloud computing es muy complejo de usar.

0,914

Formación del Personal (Amoako-Gyampah \& Salam, 2004)

(FP1) La formación recibida fue completa.

0,936

(FP3) La formación recibida me proporciona confianza en el sistema de cloud computing.

(FP4) La formación fue adecuadamente extensa y detallada.

(FP5) Los formadores eran conocedores del sistema de cloud computing y me permitían conocerlo.

Soporte y Apoyo de la Organización (Bueno \& Salmerón, 2008)

(SA1) Una persona (o dpto) está disponible para asistir en las dificultades ante el sistema.

Tabla 1 - Items y medición de la fiabilidad antes de eliminación 
se puede ver en la tabla 1, todas las cargas superan ese umbral. Para realizar un examen de la consistencia de un constructo, lo primero que debemos hacer es observar sus indicadores (ver tabla 2), concretamente el alfa Cronbach y su fiabilidad compuesta Esta evaluación mide la consistencia de un constructo en base a sus indicadores (Götz et al., 2010). El alfa de Cronbach determina un índice de consistencia para cada constructo y presenta valores comprendidos entre o y 1 . El límite inferior para aceptar la fiabilidad del constructo se suele establecer entre 0,6 y 0,7 (Hair et al., 2005). La mayor validez estará en valores próximos a 1. Respecto al otro estadístico medible para evaluar la consistencia interna: la fiabilidad compuesta o en inglés composite reliability, decir que fue propuesta por Werts et al. (1974) y que utiliza la carga de los indicadores para analizar la causalidad. Por otra parte, en cuanto a las fiabilidades compuestas, podemos afirmar que todos los constructos gozan de consistencia interna al presentar valores superiores a 0,7 (Bagozzi \& Yi, 1988; Hair et al., 2011; Nunnally, 1978). Para evaluar la validez convergente, Fornell \& Larker (1981) sugieren el uso de la varianza media extraída (AVE). La recomendación de Fornell \& Larker es que la varianza extraída media o AVE > = 0.50, lo que podemos interpretar como que más del 50\% de la varianza del constructo es debida a sus indicadores. En la tabla 2, se pueden observar los ítems utilizados y que todos los valores superan 0,5 .

\begin{tabular}{llll}
\hline Constructos & Alfa de Cronbach & $\begin{array}{l}\text { Fiabilidad } \\
\text { compuesta }\end{array}$ & AVE \\
\hline Complejidad tecnológica & 0,899 & 0,923 & 0,754 \\
Formación & 0,958 & 0,968 & 0,857 \\
Soporte y Apoyo & 0,794 & 0,906 & 0,829 \\
Intención de uso & 0,800 & 0,909 & 0,833 \\
\hline
\end{tabular}

Tabla 2 - Alfa de Cronbach y fiabilidad compuesta

En cuanto a la validez discriminante podemos decir que marca en qué medida un constructo es diferente de otros. Además, señala correlaciones de carácter débil entre constructos. Para dicho examen, se utilizan dos tipos de análisis. Por un lado, el primer análisis (ver tabla 3) consiste en chequear que la raíz cuadrada de la varianza extraída media (AVE) es mayor que la relación entre el constructo y el resto de constructos del modelo (Fornell \& Larcker, 1981).

El segundo análisis, consiste en validar la matriz de cargas cruzadas (cross loadings), donde las cargas indican las correlaciones entre los resultados de un constructo y sus indicadores. Además, debemos observar que las cargas cruzadas vienen reflejadas por las correlaciones entre las puntuaciones de un constructo y la de los indicadores de otros constructos (Henseler et al., 2009).

En cuanto a la matriz de cargas cruzadas o cross loading, decir que ahora todos los valores respectivos superan a los del mismo constructo. Se adjunta un resumen de los constructos afectados (ver tabla 4).

Hasta ahora hemos visto si el modelo cuenta con pruebas de fiabilidad y validez contrastadas. La siguiente etapa, se caracterizará por el minucioso análisis del modelo 
estructural que se llevará a cabo. En él, se contratarán las hipótesis y las relaciones entre los distintos constructos para ver si obedecen o no las relaciones establecidas en el modelo original (Ching \& Newsted, 1999, Henseler et al., 2009, 2012; Hair et al., 2012).

La explicación de la varianza se hace a partir de la cantidad de varianza de los indicadores reflectivos que es explicada por los constructos (Chin, 2010; Barclay et al., 1995). Su identificación se hace con el cálculo de los valores de $\mathrm{R}^{2} \mathrm{o}$ medida básica de cantidad de varianza del constructo que es explicada por el modelo.

La significación de los caminos se obtiene básicamente por la técnica bootstrap, que permite la obtención de valores $t$, intervalos de confianza (prueba no paramétrica) y también se pueden incluir discusiones sobre el test de Stone-Geisser (que arroja el indicador $\mathrm{Q}^{2}$ ).

\begin{tabular}{lllll}
\hline Constructos & CT & FP & IU & SA \\
\hline Complejidad Tecnológica & 0,568 & & & \\
Formación del Personal & $-0,141$ & $\mathbf{0 , 7 3 4}$ & & \\
Intención de uso & $-0,37$ & 0,453 & $\mathbf{0 , 6 9 3}$ & \\
Soporte y Apoyo & $-0,247$ & 0,705 & 0,467 & $\mathbf{0 , 6 8 7}$ \\
\hline
\end{tabular}

Tabla 3 - Correlaciones de los constructos

\begin{tabular}{lllll}
\hline Items & Complejidad Técnica & $\begin{array}{l}\text { Formación } \\
\text { Profesional }\end{array}$ & Intención de uso & $\begin{array}{l}\text { Soporte y } \\
\text { Apoyo }\end{array}$ \\
\hline CT1 & 0,647 & 0,008 & $-0,037$ & $-0,157$ \\
CT2 & 0,943 & $-0,055$ & $-0,371$ & $-0,222$ \\
CT3 & 0,922 & $-0,173$ & $-0,315$ & $-0,219$ \\
CT4 & 0,926 & $-0,184$ & $-0,363$ & $-0,249$ \\
FP1 & $-0,204$ & 0,928 & 0,468 & 0,675 \\
FP2 & 0,013 & 0,83 & 0,309 & 0,54 \\
FP3 & $-0,128$ & 0,95 & 0,425 & 0,655 \\
FP4 & $-0,144$ & 0,966 & 0,436 & 0,704 \\
FP5 & $-0,147$ & 0,948 & 0,431 & 0,672 \\
IU1 & $-0,372$ & 0,384 & 0,913 & 0,419 \\
IU2 & $-0,303$ & 0,443 & 0,913 & 0,433 \\
SA1 & $-0,288$ & 0,621 & 0,452 & 0,923 \\
SA2 & $-0,153$ & 0,668 & 0,395 & 0,898 \\
\hline
\end{tabular}

Tabla 4 - Matriz de cargas cruzadas 
Fuente: elaboración propia.(CT) Complejidad tecnológica, (FP) Formación del Personal, (IU) Intención de uso y SA (Soporte y Apoyo).

Los Niveles de $\mathrm{R}^{2}$ estudiados arrojan las siguientes conclusiones (Chin, 1998): 0,67 “ Sustancial “, o,33 “Moderado “ y o,19 “ Débil “. Como es natural, aquellas variables que no son endógenas no tienen valor $\mathrm{R}^{2}$.

Ante estos resultados debemos analizar a los autores Falk \& Miller (1992), los cuales proponen un umbral predictivo $>=0,1$. Esto explicaría que el modelo cuenta con un alto valor predictivo y su capacidad de explicar constructos endógenos. Los resultados obtenidos reflejan que el constructo Intención de Uso obtiene $\mathrm{R}^{2}=0,322$ que está en el umbral de moderado.

Los valores $\mathrm{Q}^{2}$, utilizados para calcular la relevancia predictiva del modelo (Stone, 1974; Geisser, 1975), son todos mayores que o, por lo que nuestro modelo muestra un adecuado ajuste, ya que $\mathrm{Q}^{2}=0,237$.

La evaluación se lleva a cabo mediante análisis bootstrapping en PLS para al menos 5.000 muestras y 150 casos (Hair et al., 2011). Todos los valores de los coeficientes de regresión o coeficientes $\beta$ correspondientes a las relaciones entre los constructos del modelo son $>=0,2$ (Chin, 1998) y cumplen la segunda condición, ya que cuentan con un t estadístico superior al valor 1,64 .

Concretamente, la revisión de la tabla 5 nos permite alcanzar la conclusión de que prácticamente todos los caminos son significativos. Siendo los más significativos: Complejidad Tca à Intención de Uso 10,36\% y Formación à Intención de Uso 11,95\%. Para profundizar en nuestro estudio, en la siguiente Tabla 5 se recuerdan los valores de $\mathrm{R}^{2} \mathrm{y}$ de los coeficientes $\beta$, además de mostrar las correlaciones y la varianza explicada de todas las relaciones entre constructos del Modelo.

\begin{tabular}{lllll}
\hline Constructo & $\mathbf{R}^{2}$ & $\boldsymbol{\beta}$ & Correlación & $\begin{array}{c}\text { Varianza } \\
\text { explicada }\end{array}$ \\
\hline INTENCIÓN DE USO & 0,322 & & & $32,2 \%$ \\
\hline Complejidad Técnica $\rightarrow$ Intención de Uso & $-0,280$ & $-0,370$ & $10,36 \%$ \\
\hline Formación $\rightarrow$ Intención de Uso & 0,264 & 0,453 & $11,95 \%$ \\
\hline Soporte y Apoyo $\rightarrow$ Intención de Uso & 0,212 & 0,467 & $9,9 \%$ \\
\hline
\end{tabular}

Tabla 5 - Efectos sobre las variables endógenas

\section{Discusión de los resultados}

El grado de cumplimiento de las hipótesis es si o no, es decir aceptación o no de la hipótesis plateada. Esto se hará en base a dos variables: path y estadístico t. Hay que recordar que este valor representa los coeficientes path o lo que es lo mismo: los pesos de regresión estandarizados. Ante estos resultados se puede indicar que a excepción de una hipótesis, 
todas cuentan con coeficientes $\beta$ correspondientes a las relaciones entre los constructos del modelo $>=0,2$ o presentan un t estadístico $>=1,64$. En la tabla 6, se observan los diferentes resultados de las hipótesis testadas y se confirma que son todas soportadas:

$\begin{array}{lllll}\text { No } & \text { Formulación } & \boldsymbol{\beta} & \text { Estadístico } & \text { Soportada } \\ H_{1} & \begin{array}{l}\text { La complejidad tecnológica en } \\ \text { sistemas cloud ejerce un efecto negativo } \\ \text { sobre la intención de uso }\end{array} & -0,280^{* * *} & 4,139 & \text { SI } \\ H_{2} & \begin{array}{l}\text { La formación ejerce un efecto positivo } \\ \text { sobre la intención de uso }\end{array} & 0,264^{* *} & 2,694 & \text { SI } \\ H_{3} & \begin{array}{l}\text { El soporte y apoyo ejerce un efecto } \\ \text { positivo sobre la intención de uso de los } \\ \text { sistemas cloud }\end{array} & 0,212^{*} & 1,897 & \text { SI }\end{array}$

${ }^{*} p<0$,o5 (basado en $t$ (4999), test de una cola; t-valores son: 1,6451585); **p<o,o1 (basado en $t$ (4999), test de una cola; $t$-valores son: 2,3301; ${ }^{* * * *} p<0,001$ (basado en $t$ (4999), test de una cola; $t$-valores son: 3,0984).

Tabla 6 - Cumplimiento de las hipótesis formuladas

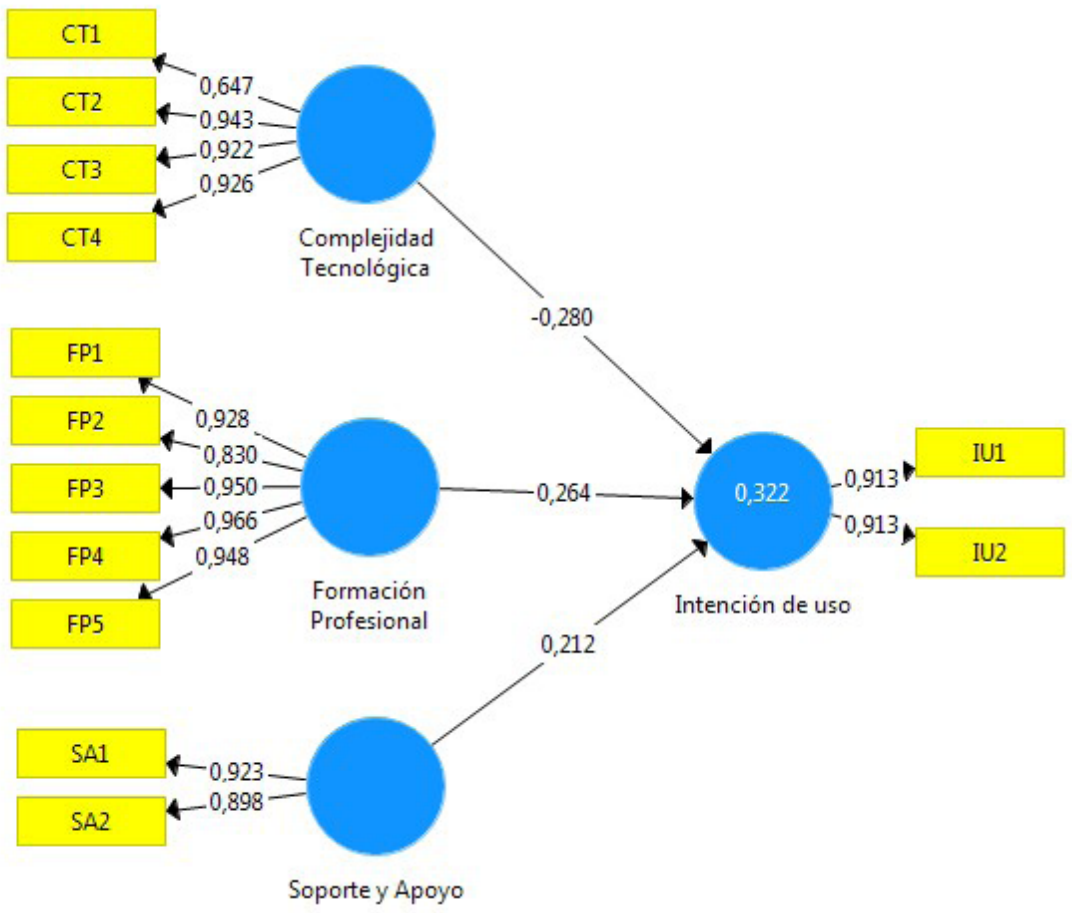

Figura 2 - Modelo final probado 


\section{Conclusiones}

Los resultados obtenidos reflejan que el modelo propuesto, basado en las variables complejidad tecnológica, formación del personal y soporte y apoyo de la organización tiende a explicar alrededor del $32,2 \%\left(\mathrm{R}^{2}=0,322\right)$ de la intención de uso del sistema cloud computing, cuyo resultado explica de manera moderada el poder predictivo del modelo en términos de la varianza explicada (Chin, 1998).

Por otra parte, las relaciones que se formulan entre estas tres variables y la intención de uso son hipótesis soportadas y estadísticamente significativas, contando, por las razones anteriormente explicadas, con un nivel predictivo moderado (Falk y Miller, 1992).

Esta investigación concluye que influye la complejidad tecnológica del sistema cloud en su intención de uso. Este resultado coincide con las conclusiones obtenidas por Tsai et al. (2010) y, dentro del ámbito específico del cloud, por el estudio de Oliveira et al. (2014). Ello implica que, aunque los sistemas cloud son percibidos como útiles y fáciles de utilizar por las organizaciones, su implantación puede suponer ciertos retos tecnológicos que algunas empresas no pueden afrontar, como, por ejemplo, la necesidad de especialistas en TIC o las exigencias técnicas para proteger procesos y datos. En cualquier caso, la influencia de la complejidad tecnológica en la Intención de uso es inversa, de modo que a mayor complejidad, la intención de uso del cloud desciende. Aunque la facilidad de uso y la utilidad han sido tradicionalmente mediadores relevantes en otros modelos de adopción, el presente trabajo pone de manifiesto que la complejidad tecnológica es también un mediador a tener en cuenta en esta nueva tecnología.

Por otra parte los servicios deberán ser fáciles de usar para cualquier usuario con una mínima formación informática a nivel usuario. La usabilidad y accesibilidad reduciría la complejidad tecnológica y aumentaría su intención de uso. Para ello, consideramos importante integrar en sistemas operativos y navegadores algunas soluciones, como ya hacen Microsoft o Google. Ahora bien, la complejidad tecnológica es clara en cuanto a su actual dificultad de comprensión o tiempo de asimilación de su aprendizaje de uso. En nuestro estudio, para los miembros de las 150 organizaciones encuestadas, la formación recibida es completa, mejora los conocimientos, proporciona confianza, contó con formadores adecuados y es adecuada desde el punto de vista de extensión y grado de detalle.

Los conocimientos en informática y las habilidades de los profesionales del sector TIC constituyen un factor esencial a tener en cuenta y como consecuencia de ello su permanente capacitación constituyen un importante desafío (Khajeh-Hosseini et al., 2012; Hutchinson et al., 2009), siendo la llamada Web 2.0 una fuente de gran importancia. La confirmación de la influencia directa de la formación del personal en la intención de uso de la tecnología, corrobora estudios previos sobre la influencia indirecta de la formación en la intención de uso, como por ejemplo a través de la percepción de utilidad (Lee at al., 2010; Gallego et al., 2015; Rajan y Baral, 2015 y, más concretamente en el ámbito cloud, (Gangwar et al., 2015), confirmando que la formación mejora la percepción de las organizaciones sobre las ventajas que los sistemas cloud provocarán en el desempeño de sus trabajadores. 
Por último, el soporte y apoyo de la organización tiene una influencia positiva en la intención de uso. Este factor es relativamente nuevo en cloud, ya que, si bien se han encontrado en otras investigaciones de sistemas de información, son demostrados empíricamente en esta investigación, en donde lo dibujamos dentro de un contexto relativamente nuevo y que se extiende con fuerza en internet: el conocimiento compartido o cooperativo. Existen pocos estudios que avalen la influencia de la formación y el soporte y apoyo en sistemas de información, más conocido como cooperación tecnológica (Bueno y Salmerón, 2008), por lo que este trabajo constituye una aportación en un factor de influencia novedoso en los servicios cloud computing, que a la vez ha sido contrastado con la complejidad tecnológica.

Algunos aspectos que podrían ser objeto de futuras líneas de investigación y complementarios a la investigación realizada, podrían ser el estudio de los factores que condicionan la adopción de la tecnología cloud desde la perspectiva de la cooperación empresarial, para así evaluar el efecto que tendría la participación en redes empresariales destinadas a aumentar la capacitación organizativa y el reciclaje del personal técnico en la adopción de los sistemas cloud.

\section{Referencias}

ACKERMANN, T., WIDJAJA, T., BENLIAN, A., \& BUXMANN, P. (2013). Perceived IT security risks of cloud computing: Conceptualization and scale development. In Proceedings of the 33rd International Conference on Information Systems (ICIS 2012). Orlando, FL, US.

ALSHAMAILA, \&., PAPAGIANNIDIS, S., \& LI, F. (2013). Cloud computing adoption by SMEs in the north east of England. A multi-perspective framework. Journal of Enterprise Information Management, 26(3), 250-275.

AMOAKO-GYAMPAH, K., \& SALAM, A. (2004). An extension of the technology acceptance model in an implementation environment. Information \& Management, 41(6), 731-745.

BAGOZZI, R., \& YI, Y. (1988). On the evaluation of structural equation models. Journal of the Academy of Marketing Science, 16(1), 74-94.

BARCLAY, D., HIGGINS, C., \& THOMPSON, R. (1995). The Partial Least Squares (PLS) Approach to Causal Modelling: Personal Computer Adoption and Use as an Illustration. Technology studies, 2(2), 285-309.

BENLIAN, A., HESS, T., \& BUXMANN, P. (2009). Drivers of saas-adoption na empirical study of different application types. Business Information System Engineering, 1, 357-369.

BENLIAN, A., KOUFARIS, M., \& HESS, T. (2011). Service quality in software-asa-service: Developing the SaaS-qual measure and examining its role in usage continuance. Journal of Management Information Systems, 28(3), 85- 126. 
BRANDT, T., TIAN, Y., \& HEDWIG, M. (2012). Autonomic management of software as a service systems with multiple quality of service classes. In Proceedings of the 2oth European Conference on Information Systems (ECIS). Barcelona, Spain.

BURDA, D., \& TEUTEBERG, F. (2014). The role of trust and risk perceptions in cloud archiving. Journal of High Technology Management Research, 25(2), 172-187.

BUENO, S., \& SALMERÓN, J. (2008). TAM-based success modeling in ERP. Interacting with Computers, 20(6), 515-523.

BUYYA, R., YEO, C., VENUGOPA, S., BROBERG, J., \& BRANDIC, I. (2009). Cloud computing and emerging it platforms: vision, hype, and reality for delivering computing as the $5^{\text {th }}$ utility. Future Generation Computer Systems, 25(6), 599-616.

CARMINES, E. G., \& ZELLER, R. (1979). Reliability and Validity Assessment. Newbury Park, CA: Sage Publications.

CEPEDA, G., \& ROLDÁN, J. (2004). Aplicando en la práctica la Técnica PLS en la Administración de Empresas. In Procedimentos de XIV Congreso Anual de ACEDE. Murcia, España.

CHEUNG, R., \& VOGEL, D. (2013). Predicting user acceptance of collaborative technologies: An extension of the technology acceptance model for e-learning. Computers y Education, 63, 160-175.

CHIN, W. W. (1998). The partial least squares approach to structural equation modeling. Modern methods for business research, 295(2), 295-336.

CHIN W. (2010). How to write up and report PLS analyses. In Vinzi, V. E., Chin, W.W., Henseler, J., \& Wang, H. (Eds.). Handbook of Partial Least Squares: concepts, methods and applications in marketing and related Fields. (pp. 655-690). Berlin: Springer.

CHING, W., \& NEWSTED, P. (1999). Structural Equation modeling. Analysis with small samples using partial least squares. Statistical strategies for small sample research, 1(1) 307-341.

COOPER R.B., \& ZMUD R.W. (1990). Information technology implementation research: a technological diffusion approach. Management Science, 36(2): 123-139.

FALK, R., \& MILLER, N. (1992). A Primer for Soft Modeling. Akron, OH: University of Akron Press.

FEUERLICHT, G., \& GOVARDHAN, S. (2010). Impact of cloud computing: beyond a technology trend. In The Proceedings of the International Conference on Systems Integration (Oeconomica), June 8-9. (pp. 1-8). Prague.

FEUERLICHT, G., BURKON, L., \& SEBESTA, M. (2011). Cloud computing adoption: what are the issues? System Integration, 18(2), 187-192. 
FISHBEIN, M., \& AJZEN, I. (1975). Belief, Attitud, Intention, and Behavior: An Introduction to Theory and Research. Reading, MA: Addison-Wesley.

FORNELL, C. (1982). A Second Generation of Multivariate Analysis: Methods. Measurement and evaluation (Vol. 2). Westport, CT: Praeger Publishers.

FORNELL, C., \& LARCKER, D. F. (1981). Evaluating structural equation models with unobservable variables and measurement error. Journal of Marketing Research, 18(1), 39-50.

FORRESTER RESEARCH. (2009). Global IT Market Outlook: 20o9. The Global Recessions Will Slow IT Purchases Growth To A Crawl. Retrieved from: https:// www.forrester.com/report/Global+IT+Market+Outlook+2009/-/E-RES46676

FORTES, N., PEREIRA, J. H., \& COSTA, J. F. D. (2016). A adoção de serviços cloud computing pelas empresas portuguesas: O papel dos esforços de marketing. RISTI - Revista Ibérica de Sistemas e Tecnologias de Informação, (18), 33-48.

FROST \& SULLIVAN. (Diciembre 2008). Market Demands for Hosted UC Services. San Antonio, TX: Frost \& Sullivan.

GALLEGO, M.D., BUENO, S., RACERO, F.J. \& NOYES, J. (2015). Open source software: The effects of training on acceptance. Computers in Human Behavior 49, 390-399.

GANGWAR, H., DATE, H., \& RAMASWAMY, R. (2015). Understanding determinants of cloud computing adoption using an integrated TAM-TOE model. Information Technology, Journal of Entreprise Information, 28(1), 107-130.

GARTNER. (2008). Assessing the Security Risks of Cloud Computing. Retrieved from: https://www.gartner.com/doc/685308/assessing-security-risks-cloud-computing

GÉCZY, P., IZUMI, N., \& KÔITI, H. (2012). Cloudsourcing: managing cloud adoption. Global Journal of Business Research, 6(2), 57-70.

GEISSER, S. (1975). The predictive sample reuse method with applications. Journal of the American Statistical Association, 70(350), 320-328.

GÖTZ, O., LIEHR-GOBBERS, K., \& KRAFFT, M. (2010). Evaluation of structural equation models using the Partial Least Squares (PLS) approach. In Vinzi, W. W. V. E. (ed.) Handbook of partial least squares, (pp. 691-711). Berlin: Springer.

GROVER V. (1993) Empirically derived model for the adoption of customer-based interorganizational systems. Decision Sciences, 24(3):603-39.

GUPTA, P., SEETHARAMANA, A., \& RAJB, J. (2013). The usage and adoption of cloud computing by small and medium businesses. International Journal of Information Management, 33, 861-874

HAIR, J., BLACK, W., BABIN, B., ANDERSON, R., Y TATHAM, R. (2005). Multivariate data analysis (5th ed.). Upper Saddle River, NJ: Prentice Hall.

HAIR, J. F., RINGLE, C. M., \& SARSTEDT, M. (2011). PLS-SEM: Indeed a silver bullet. Journal of Marketing Theory and Practice, 19(2), 139-151. 
HAIR, J., MARKO, S., RINGLE, C., \& MENA, J. (2012). An assessment of the use of partial least squares structural equation modeling in marketing research. Journal of the Academy Markenting Science 40(3), 414-433

HENSELER, J., RINGLE, C. M., \& SINKOVICS, R. R. (2009). The Use of Partial Least $\mathrm{S}$ quares Path Modeling in International Marketing. Advances in International Marketing (20), 277-320.

ION, I., SACHDEVA, N., KUMARAGURU, P., \& CAPKUN, S. (2011). Home is safer than the cloud!: Privacy concerns for consumer cloud storage. In Proceedings of the 7 th Symposium on Usable Privacy and Security. Pittsburgh, Pennsylvania.

JOO, J. \& SANG, \&. (2013). Exploring Koreans' smartphone usage: An integrated model of the technology acceptance model and uses and gratifications theory. Computers in Human Behavior, 29(6), 2512-2518.

KHAJEH-HOSSEINI, A., GREENWOOD, D., SMITH, J., \& SOMMERVILLE, I. (2012). The cloud adoption toolkit: supporting cloud adoption decisions in the enterprise. Software: Practice and Experience 42(4), 447-465.

LEE, D., \& LEHTO, M. (2013). User acceptance of YouTube for procedural learning: An extension of the Technology Acceptance Model. Computers \& Education 61, 193-208.

LEE, D.H., LEE, S.M., OLSON, D.L., \& CHUNG, S.H. (2010). The effect of organizational support on ERP implementation. Industrial Management y Data Systems, 110(12), 269-283.

LIN, W. B., WANG, M. K., \& HWANG, K. P. (2010). The combined model of influencing on-line consumer behavior. Expert Systems with Applications, 37(4), 3236-3247.

LIN, A., \& CHEN, N. (2012). Cloud computing as an innovation: Percepetion, attitude, and adoption. International Journal of Information Management, 32(6), 533-540.

LOW, C., CHEN, Y., \& WU, M. (2011). Understanding the determinants of cloud computing adoption. Industrial Management \& Data Systems, 111(7), 1006-1023.

MARTENS, B. \& TEUTEBERG, F., (2012). Decision-making in cloud computing environments: A cost and risk based approach. Information Systems Frontiers, 14(4), 871-893.

MELL, P., \& GRANCE, T. (2011). Definition of cloud computing. Accedido el 12 de Octubre de 2016 en http://www.nist.gov/itl/cloud/

MESSERSCHMIDT, C. H. (2013). Explaining the adoption of grid computing: An integrated institutional theory and organizational capability approach. Journal of Strategic Information Systems, 22(2), 137-156.

NUNNALLY, J. (1978). Psychometric Theory. New York: McGraw-Hill.

OLIVEIRA, T., \& MARTINS, M. (2010). Understanding e-business adoption across industries in European countries. Industrial Management Data System, 110, 1337-1354. 
OLIVEIRA, T., THOMAS, M., \& ESPADANAL, M. (2014). Assessing the determinants of cloud computing adoption: An analysis of the manufacturing and services sectors. Information \& Management, 51(5), 497-510.

PALOS, P.R., \& CORREIA, M.B. (2016). La actitud de los recursos humanos de las organizaciones ante la complejidad de las aplicaciones SaaS. Dos Algarves: A Multidisciplinary e-Journal, (28), 87-103. doi:10.18089/DAMeJ.2016.28.6

PALOS P.R., \& CORREIA, M. (2017). The Paradigm of the Cloud and Web Accessibility and its Consequences in Europe. In Proceedings of the 7 th International Conference on Software Development and Technologies for Enhancing Accessibility and Fighting Info-exclusion (DSAI 2016). ACM. Vila Real, Portugal. doi:10.1145/3019943.3019995

PALOS-SÁNCHEZ, P. R., ARENAS-MÁRQUEZ, F. J., \& AGUAYO-CAMACHO, M. (2017a). Determinants of Adoption of Cloud Computing Services by Small, Medium and Large Companies. Journal of Theoretical \& Applied Information Technology, 95(6) 1273.

PALOS-SANCHEZ, P. R., ARENAS-MARQUEZ, F. J., \& AGUAYO-CAMACHO, M. (2017b). Cloud Computing (SaaS) adoption as a strategic technology: Results of an empirical study. Mobile Information Systems, 2017. Retrieved from https://www. hindawi.com/journals/misy/aip/2536040/

PEARSON, S. (2011). Toward accountability in the cloud. IEEE Computer Society, 15(4), 64-69.

PREMKUMAR, G., \& ROBERTS, M. (1999). Adoption of new information technologies in rural small businesses. Omega, 27(4), 467-484.

RAJAN, C.A. \& BARAL, R. (2015). Adoption of ERP system: An empirical study of factors influencing the usage of ERP and its impact on end user. IIMB Management Review, 27(2), 105-117.

ROGERS, E. M., \& SHOEMAKER, F. F. (1971). Communication of Innovations; A CrossCultural Approach. New York: Free press.

SÁ, F., \& ROCHA, Á. (2012). Definição da arquitetura de informação em organismo da administração pública local. RISTI - Revista Ibérica de Sistemas e Tecnologias de Informação, (10), 51-64.

SCHILL, A. (2011). Information dispersion over redundant arrays of optimal cloud storage for desktop top users. In Proceedings of the 4th IEEE International Conference on Utility and Cloud Computing (UCC). Melbourne, Australia.

SCHILLEWAERTA, N., AHEARNEB, M. J., FRAMBACHC, R. T., \& MOE-NAERTD, R. K. (2005). The adoption of information technology in the sales force. Industrial Marketing Management, 34(4), 323-336.

SENK, C. (2013). Adoption of security as a service. Journal of Internet Services and Applications, 4, 11. 
SOMERS, T., \& NELSON, K. (2003). The impact of strategy and integration mechanisms on enterprise system value: empirical evidence from manufacturing firms. European Journal of Operational Research, 146(2), 315-338.

SON, H., PARK, Y., KIM, C., \& CHOU, J. (2012). Toward an understanding of construction professionals' acceptance of mobile computing devices in South Korea: An extension of the technology acceptance model. Automation in Construction 28, 82-90.

SON, J. Y., \& BENBASAT, I. (2007). Organizational buyers' adoption and use of B2B electronic marketplaces: efficiency-and legitimacy-oriented perspectives. Journal of management information systems, 24(1), 55-99.

SPILLNER, J., BOMBACH, G., MATTHISCHKE, S., MULLER, J., TZSCHICHHOLZ, R., \& STONE M. (1974). Cross-validatory choice and assessment of statistical predictions. Journal of the Royal Statistical Society Series B 36, 1974, 111-133.

TAYLOR, S., \& TODD, P. A. (1995). Understanding information technology usage: A test of competing models. Information systems research, 6(2), 144-176.

TORRES, J. (2011). Empresas en la Nube. Ventajas y retos del Cloud Computing. Barcelona: Libros de Cabecera.

TSAI, M., LEE, W. \& WU, H. (2010). Determinants of RFID adoption intention: evidence from Taiwanese retail chains. Information Management, 47, 255-261.

VAQUERO, L. M., RODERO-MERINO, L., CACERES, J., \& LINDNER, M. (2009). A break in the clouds: Towards a cloud definition. Computer Communication Review, 39(1), 50-55.

VILE, A., \& LIDDLE, J. (2009). The Savvy guide to HPC, grid, data grid, virtualization and cloud computing. The Savvy Guide To Ltd.

VOUK, M. (2008). Cloud computing - issues, research and implementations,. Journal Computer Information Technology, 16, 235- 246.

WALTERBUSCH, M., MARTENS, B., \& TEUTEBERG, F. (2013). Evaluating cloud computing services from a total cost of ownership perspective. Management Research Review, 36(6), 613-638.

WANG, C., CHOW, S., WANG, Q., REN, K. \& LOU, W., (2013). Privacy-preserving public auditing for secure cloud storage. IEEE Transactions on Computers, 62(2), $362-375$.

WERTS, C. E., LINN, R. L. \& JÖRESKOG, K. G. (1974). Intraclass reliability estimates: Testing structural assumptions. Educational and Psychological measurement, 34(1), 25-33.

WU, W. W. (2011). Developing an explorative model for SaaS adoption. Expert systems with applications, 38(12), 15057-15064.

WU, W.-W., LAN, L., \& LEE, Y.-T. (2011). Exploring decisive factors affecting an organization's SaaS adoption: a case study. International Journal Information Management, 31, 556-563. 